interesting phenomena - for example, the quantum correlations that are expected to be exhibited by pairs of sound waves produced at the event horizon. -

Silke Weinfurtner is at the School of Mathematical Sciences, University of Nottingham, Nottingham NG7 2RD, UK. e-mail:silke.weinfurtner@nottingham.ac.uk

1. Hawking, S. W. Nature 248, 30-31 (1974).

2. Unruh, W. G. Phys. Rev. D 14, 870-892 (1976)

3. de Nova, J. R. M., Golubkov, K., Kolobov, V. I. \&
Steinhauer, J. Nature 569, 688-691 (2019).

4. Unruh, W. G. Phys. Rev. Lett. 46, 1351-1353 (1981).

5. Barceló, C., Liberati, S. \& Visser, M. Living Rev. Relativ. 14, 3 (2011)

6. Weinfurtner, S., Tedford, E. W., Penrice, M. C. J., Unruh, W. G. \& Lawrence, G. A. Phys. Rev. Lett. 106 021302 (2011).

7. Euvé, L.-P., Michel, F., Parentani, R., Philbin, T. G. \& Rousseaux, G. Phys. Rev. Lett. 117, 121301 (2016).

8. Garay, L. J., Anglin, J. R., Cirac, J. I. \& Zoller, P. Phys. Rev. Lett. 85, 4643-4547 (2000).

9. Lahav, O. et al. Phys. Rev. Lett. 105, 240401 (2010).

10.Steinhauer, J. Nature Phys. 12, 959-965 (2016).

11. Coutant, A. \& Weinfurtner, S. Phys. Rev. D 97 025006 (2018)

\title{
Guarding the gate for mitochondrial entry
}

\section{The protein-import systems of organelles can become clogged by proteins. A protein from one organelle, the endoplasmic reticulum, is found to also unclog such blockages in mitochondrial organelles. SEE ARTICLE P.679}

\section{SYLVIE CALLEGARI \& PETER REHLING}

$\mathrm{O}$ rganelles known as mitochondria are the energy-generating powerhouses of the cell. As a crucial part of the cellular machinery, disruption to their function could have serious consequences, and so mechanisms exist to combat mitochondrial dysfunction. On page 679, Mårtensson et al. ${ }^{1}$ report a pathway that tackles problems concerning the import of proteins into mitochondria, as they arise, to prevent mitochondrial and cellular dysfunction. This pathway uses part of the cellular repair kit that handles damaged proteins in another organelle - the endoplasmic reticulum (ER).

At least 1,000 proteins reside within the mitochondria of yeast, where they carry out functions that contribute to cellular energy production and metabolism ${ }^{2}$. Almost all of these proteins are made in the cytoplasm and are then transported into mitochondria in an unfolded precursor form. Mitochondria receive a heavy inbound flow of protein traffic, and the entry route that bears the brunt of this influx is a protein complex on the mitochondrial surface called the TOM complex. When the TOM complex becomes overloaded or is compromised due to mitochondrial damage, unfolded mitochondrial proteins can accumulate in the cytoplasm and cause cellular toxicity $^{3-6}$. Mechanisms exist to combat the stress to cells that results from this ${ }^{3-6}$, but how mitochondria handle protein-import failure as it arises was unclear.

The pore of a TOM complex will sometimes become clogged with a mitochondrial protein (Fig. 1). Protein misfolding is a common cellular event that can be exacerbated by stress or occur if a protein is in a mutant form ${ }^{7}$. A mitochondrial precursor protein that folds prematurely in the pore of the TOM complex could become stuck there. A blockage might also occur if the energy levels of the cell drop, leading to a slowing or stalling of protein import ${ }^{8}$. If such an obstruction is not removed, the accumulation of unfolded precursor proteins in the cytoplasm can trigger transcriptional changes that lead to the unleashing of a cellular stress response ${ }^{4}$. Such stress-response pathways ${ }^{3,4}$ include the UPRam pathway and the mitoCPR pathway, which prime the cytoplasmic waste-disposal system - a protein complex called the proteasome - to degrade the accumulated precursor proteins.

Mårtensson et al. investigated whether a system is in place to constantly monitor the TOM complex and to remove trapped precursor proteins, thereby preventing blockages from hampering protein import to an extent that causes cellular stress. Such a monitoring system is in place for an entry gate into the ER ${ }^{9}$. To identify possible factors that might clear away trapped precursor proteins, Mårtensson $e t$ al. purified the TOM complex from the mitochondria of yeast cells. One of the TOM-complex-associated proteins that they identified was Ubx2, which was a surprise. This is because Ubx2 is best known for its activity at the ER, where it functions in the routine clearance of misfolded proteins as part of an ER-specific quality-control pathway called ER-associated degradation (ERAD) ${ }^{10,11}$. Mårtensson and colleagues conducted biochemical experiments that showed that Ubx2 exists in two distinct cellular pools, one at the ER and another on the mitochondrial surface

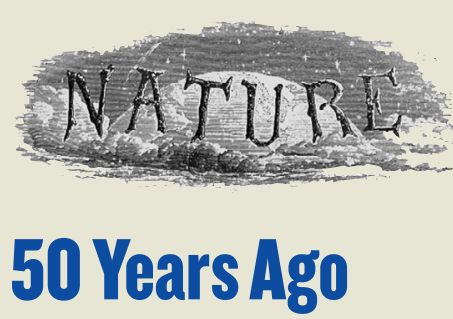

Apollo 10 has demonstrated the technical virtuosity of NASA and its works. It is hard now to remember that it is merely two years since an unlucky fire cost the lives of three astronauts at Cape Kennedy. Then it seemed as if the dream of getting to the Moon before the end of the sixties would be broken. Now, as the numbers tick by towards eleven, the meticulous care with which the whole enterprise has been conducted becomes increasingly apparent. The Apollo programme seems to be bursting with a host of technological sagas ... It is true that the United States is hoping that there will flow from this accumulation of expertise a further refinement of the skill ... which has made it the inventive place it is already ... But the more lasting achievement is the way in which the anticipation of technical problems has created a sense of mastery of machines ... The old cliché of the returning hero that it is the team which deserves the honours has become a truism. From Nature 31 May 1969

100 Years Ago

As the result of an executive order issued by President Wilson ... the temporary arrangement inaugurated two years previously has acquired permanence as the National Research Council of the United States. The history of this organisation is instructive in showing that in time of national stress the Governmental authorities appreciate the necessity for active co-operation from scientific bodies or individuals who have in peace conditions received but little recognition or support ... Thus it is that the ... direction of research work in the United States has become vested in a body of men whose ... work during the war ... has shown that they are competent to handle the great problems which go with peace and reconstruction. From Nature 29 May 1919 


\section{RESEARCH NEWS \& VIEWS}

\section{nature research}

\section{ACADEMIES}

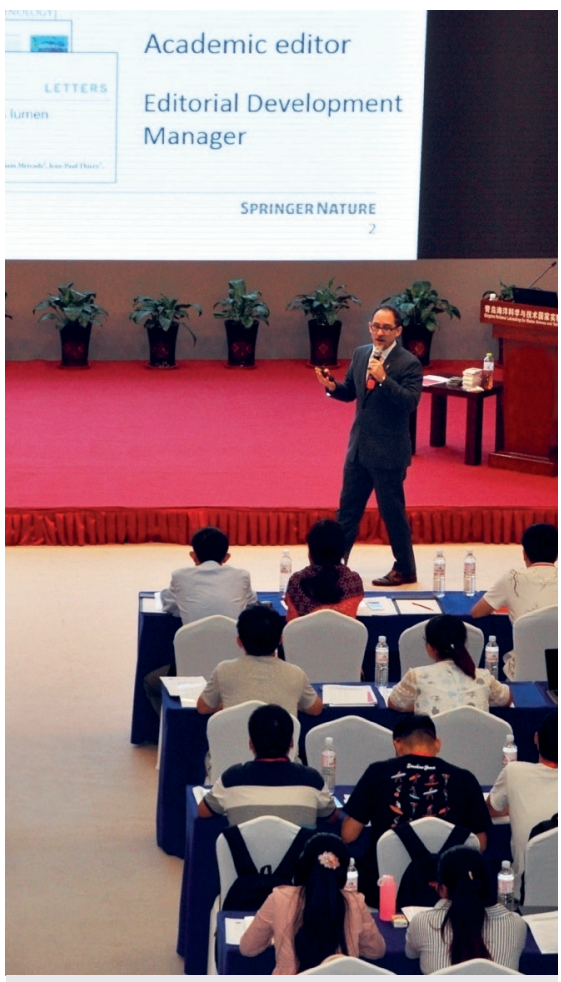

Training workshops

for researchers

A series of workshops

to support researchers,

covering topics such as

getting published, journal

editing, clinical research

methodology, and

applying for research

positions.

\section{Visit}

partnerships.nature. com/academies to host an academy at your institution.

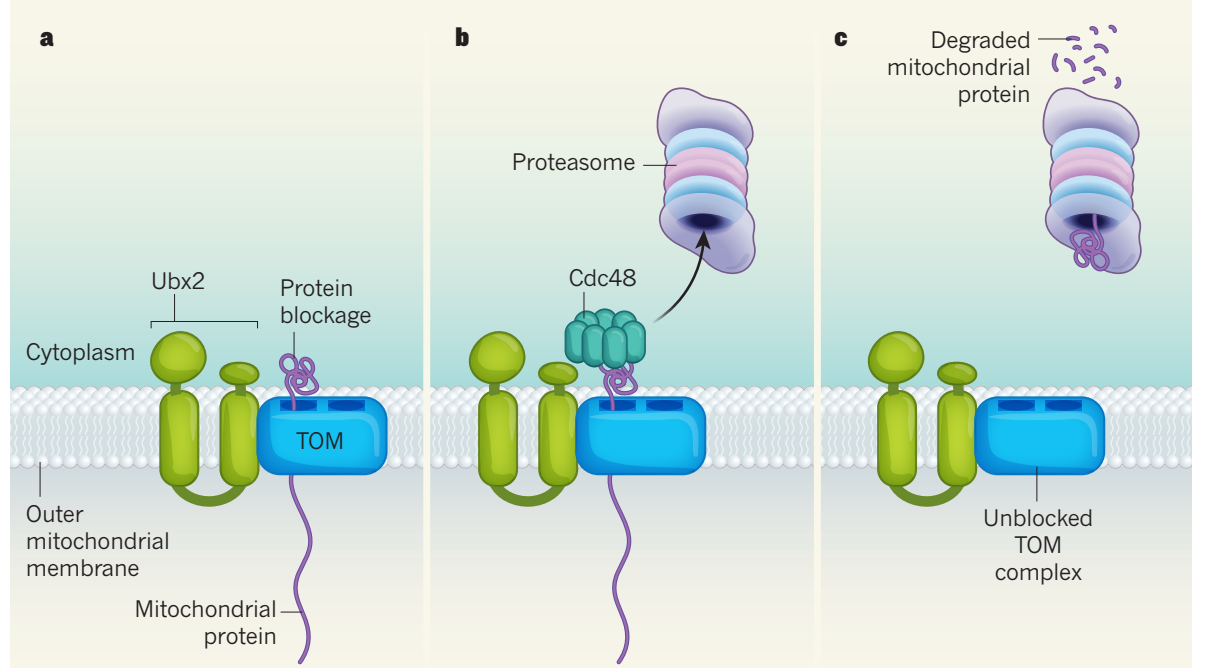

Figure 1 | A mechanism for unclogging blockages in the protein-import system of mitochondria. a, Unfolded mitochondrial proteins are imported from the cytoplasm into mitochondria through a protein complex called TOM that is located on the outer mitochondrial membrane. However, the TOM complex can become blocked when, for example, a protein folds prematurely as it passes through the complex. Mårtensson et al. ${ }^{1}$ report a system in yeast cells that monitors and unclogs such blockages. The protein Ubx2, which acts in quality-control systems for another organelle (the endoplasmic reticulum $)^{10,11}$, is required to recognize protein blockages in the TOM complex. b, Ubx2 recruits the protein $\mathrm{Cdc} 48$, which removes the clogged protein and delivers it to a protein-degradation complex called the proteasome. $c$, The degradation of the mitochondrial protein completes the process that unclogs the TOM complex. Figure based on Fig. $4 \mathrm{f}$ of ref. 1.

that is associated with TOM complexes.

In the ERAD pathway, enzymes add the protein ubiquitin to misfolded proteins that are trapped in the $\mathrm{ER}^{10,11}$. This ubiquitin tag is recognized by Ubx2, which triggers the recruitment of a protein called Cdc48 - a type of enzyme called an AAA ATPase. Cdc48 extracts misfolded proteins from the ER and delivers them to the proteasome for degradation $^{10,11}$. To ascertain whether Ubx2 might function in mitochondria in a similar way, Mårtensson and colleagues tested whether Cdc48 interacts with the TOM complex. They found that it does, and also that the interaction depends on Ubx2. When the authors blocked the energy source that is needed for mitochondrial protein import, this resulted in an accumulation of precursor proteins only in mitochondria that lacked Ubx2.

Precursor-protein accumulation in the absence of Ubx2 was also observed if mitochondrial protein import was slowed by an alternative means - the use of a mutant version of a protein that drives protein import into mitochondria ${ }^{12,13}$. In this set-up, Mårtensson et al. observed that precursor proteins accumulated in the cytoplasm of cells that had defects in Cdc48 or in a component of the proteasome, which indicates that $\mathrm{Cdc} 48$ and the proteasome have a key role in this pathway.

To specifically determine whether Ubx2 is needed to remove precursor proteins that are clogging the TOM complex, Mårtensson and colleagues expressed mitochondrial precursor proteins, in yeast, that were chosen because they are imported slowly into the organelle. The authors engineered this scenario to boost the number of blockage events, and then extracted TOM complexes. They found that, in cells that lacked Ubx2, precursor proteins were stuck in TOM complexes. However, in cells where Ubx2 was present, no precursor proteins were detected in TOM complexes. This key piece of evidence indicates that Ubx2 is part of a previously unknown quality-control mechanism that surveils the TOM complex, and Marrtensson et al. have named it the mitochondrial protein translocation-associated degradation (mitoTAD) pathway.

Mårtensson and colleagues' findings raise two crucial questions. First, what is the relationship between the mitoTAD pathway and the mitochondrial quality-control pathways that operate under conditions of cellular stress? During their investigation, the authors unearthed a functional link between Ubx2 and the protein Vms1, as well as with the enzyme Msp1 (which, like Cdc48, is an AAA ATPase). Both Vms1 and Msp1 have been implicated in mitochondrial qualitycontrol processes ${ }^{14-17}$. When the authors removed these proteins from cells that lacked Ubx2, mitochondria lost their energyproducing function and ubiquitin-tagged proteins accumulated. This result suggests that Vms1 and Msp1 work in a way that is complementary to Ubx2 function. An investigation to assess the crosstalk between these pathways and the mitoTAD pathway might deepen our understanding of mitochondrial quality-control mechanisms. 
Second, how does Ubx2 recognize that a precursor protein is stuck in the TOM complex? Ubx 2 can bind to ubiquitin, so ubiquitin is probably used to tag clogged precursor proteins. Defining the components of the ubiquitination machinery and the ubiquitin signals that govern not just the mitoTAD pathway, but also the stress-induced quality-control pathways that regulate mitochondrial protein import will be an important direction for future research. The fact that the ER and mitochondria use the same protein for quality control might imply that these processes are co-regulated.

Many other fascinating questions also remain to be addressed. In the meantime, Mårtensson and colleagues' work has painted an illuminating picture of a previously unknown aspect of how mitochondria protect their main entry gate.

Sylvie Callegari and Peter Rehling are in

the Department of Cellular Biochemistry,

University Medical Center Göttingen, 37073

Göttingen, Germany. P.R. is also at the Max

Planck Institute for Biophysical Chemistry,

Göttingen.

e-mail:peter.rehling@medizin.uni-goettingen.de

1. Mårtensson, C. U. et al. Nature 569, 679-683 (2019).

2. Pfanner, N., Warscheid, B. \& Wiedemann, N. Nature Rev. Mol. Cell Biol. 20, 267-284 (2019).

3. Wrobel, L. et al. Nature 524, 485-488 (2015).

4. Weidberg, H. \& Amon, A. Science 360, eaan4146 (2018).

5. Boos, F. et al. Nature Cell Biol. 21, 442-451 (2019).

6. Wang, X. \& Chen, X. J. Nature 524, 481-484 (2015).

7. Gregersen, N., Bross, P., Vang, S. \& Christensen,
J. H. Annu. Rev. Genomics Hum. Genet. 7, 103-124 (2006).

8. Mehnert, C. S. et al. J. Biol. Chem. 289, 27352-27362 (2014).

9. Ast, T., Michaelis, S. \& Schuldiner, M. Cell 164 103-114 (2016).

10.Neuber, O., Jarosch, E., Volkwein, C., Walter, J. \& Sommer, T. Nature Cell Biol. 7, 993-998 (2005).

11.Schuberth, C. \& Buchberger, A. Nature Cell Biol. 7, 999-1006 (2005).

12.van der Laan, M. et al. Mol. Cell Biol. 25, 7449-7458 (2005).

13.Schendzielorz, A. B. et al. J Cell Bio/ 216, 83-92 (2017).

14.Izawa, T., Park, S.-H., Zhao, L., Hartl, F. U. \& Neupert, W. Cell 171, 890-903 (2017)

15. Heo, J.-M. et al. Mol. Cell 40, 465-480 (2010).

16.Okreglak, V. \& Walter, P. Proc. Natl Acad. Sci. USA 111, 8019-8024 (2014).

17.Chen, Y.-C. et al. EMBO J. 33, 1548-1564 (2014).

This article was published online on 22 May 2019.

\section{Brain cells invade prostate tumours}

Prostate cancer contains nerve cells that are linked to disease progression, but their source was unknown. A mouse study reveals that cells from the brain invade prostate tumours and give rise to this nerve-cell population. SEE ARTICLE P.672

\section{SIMON T. SCHAFER \& FRED H. GAGE}

$\mathrm{M}$ any studies ${ }^{1-6}$ in the past decade have shown that interactions between tumour cells and nerve cells can influence the progression of various types of cancer. In prostate tumours, the presence of newly formed nerve cells is associated with the progression of malignancy and tumour spread to other locations in the body ${ }^{1,2,7}$. The microenvironment of a tumour can permit the development of such new nerve cells in a process called neo-neurogenesis. Yet how these nerve cells emerge in tumours in the first place has been a key unanswered question. Writing in Nature, Mauffrey et al. ${ }^{8}$ report a study in mice which indicates that a type of precursor cell called a neural progenitor cell, from a region of the brain in which the normal process of nerve-cell formation (neurogenesis) occurs, migrates to the site of prostate cancer. These cells invade the tumour microenvironment and give rise to new nerve cells.

The processes by which embryos and solid tumours develop are thought to share many features. In most multicellular animals, the nervous system and the vasculature have roles in both of these processes. In mice, neurons arise throughout life, by neurogenesis from neural progenitor cells in two regions of the adult brain - the subventricular zone and the dentate gyrus ${ }^{9}$. Although there has been controversy lately about the extent of neurogenesis

in adult humans ${ }^{10-12}$, decades of previous work have indicated that humans generate new neurons throughout the course of life only in the dentate gyrus ${ }^{13,14}$.

To address the origin of nerve cells in prostate tumours, Mauffrey and colleagues used a mouse model of prostate cancer called Hi-myc. The authors report that prostate tumours in these animals were associated with a type of newborn nerve cell called a neuroblast (Fig. 1). These cells express the protein doublecortin

"Neural progenitor cells from the subventricular zonecan migrate from the brain." (DCX), which is also expressed by special types of neural progenitor cell, which give rise to neurons.

The authors isolated DCX-expressing cells (which might be neural progenitor cells or neuroblasts) from samples of the animals' prostate tumours, and analysed the expression of genes and proteins. Using an approach termed antigenic profiling, they tracked the source of the DCX-expressing neuroblast cells that they had isolated, and found that the cells expressed several proteins characteristic of neural progenitor cells that can give rise to neurons. The neuroblasts did not express the gene $M y c$, which drives prostate tumour formation in the Hi-myc model, leading the authors to conclude that these neuroblasts were probably not derived from tumour cells. Further neuroblast-cell profiling did not reveal any obvious similarities to other types of cell in the tumour microenvironment, such as immune or endothelial cells. The authors found that the tumour-associated neuroblasts had molecular hallmarks similar to those of neural progenitor cells from the subventricular zone. Moreover, the cells could differentiate into neuronal-like cells when grown in vitro.

Mauffrey and colleagues next studied neural progenitor cells in the mouse brain, obtaining crucial support for their proposal that these cells drive neo-neurogenesis in prostate tumours. The authors tracked the cells using a technique that labels neural progenitor cells in specific brain regions. This revealed that neural progenitor cells from the subventricular zone can migrate from the brain and travel through blood vessels to specifically invade prostate tumours. However, the molecular factors and the mechanisms that guide the migration of these cells to this location are unknown. The authors found no evidence that neural progenitor cells from the dentate gyrus exited the brain. And they report that there was a selective breach in the blood-brain barrier around the region of the subventricular zone, but that no such breach was associated with the dentate gyrus.

To determine the relevance of their findings to other types of cancer, Mauffrey and colleagues used various mouse cancer models generated through the transplantation of human tumour cells. The authors consistently observed a similar mode of neural-progenitorcell migration from the brain to the tumours. Moreover, when they used a technique to produce mice that had selective depletion of DCX-expressing neural progenitor cells and neuroblasts, the resulting animals showed impaired tumour establishment compared with control mice. When mice bearing human prostate tumours were transplanted with neuroblasts from either mouse prostate 